IJMMS 26:11 (2001) 649-669

PII. S0161171201020154

http://ijmms.hindawi.com

(c) Hindawi Publishing Corp.

\title{
ORBIFOLDS AND FINITE GROUP REPRESENTATIONS
}

\author{
LI CHIANG and SHI-SHYR ROAN
}

(Received 12 December 2000)

\begin{abstract}
We present our recent understanding on resolutions of Gorenstein orbifolds, which involves the finite group representation theory. We concern only the quotient singularity of hypersurface type. The abelian group $A_{r}(n)$ for $A$-type hypersurface quotient singularity of dimension $n$ is introduced. For $n=4$, the structure of Hilbert scheme of group orbits and crepant resolutions of $A_{r}(4)$-singularity are obtained. The flop procedure of 4 -folds is explicitly constructed through the process.
\end{abstract}

2000 Mathematics Subject Classification. 20Cxx, 14Jxx, 14Mxx.

1. Introduction. It is well known that the theory of "minimal" resolutions of singularity of algebraic (or analytical) varieties differs significantly when the (complex) dimension of the variety is larger than two. As the prime achievement in algebraic geometry of the 1980s, the minimal model program in the 3-dimensional birational geometry carried out by Mori and others, has provided an effective tool for the study of algebraic 3-folds (see [17] and the references therein). Meanwhile, Gorenstein quotient singularities in dimension 3 has attracted considerable interests among geometers due to the development of string theory, by which the orbifold Euler characteristic of an orbifold was proposed as the vacuum description of models built upon the quotient of a manifold [5]. The consistency of physical theory then demanded the existence of crepant resolutions which are compatible with the orbifold Euler characteristic. The complete mathematical justification of the conjecture was obtained in the mid-90s (see [26] and the references therein). However, due to the computational nature of methods in the proof, the qualitative understanding of these crepant resolutions has still been lacking on certain aspects from a mathematical viewpoint. Until very recently, by the development of Hilbert scheme of a finite group $G$-orbits, initiated by Nakamura and others with the result obtained in [1, 6, 10, 11, 12, 18, 19] it strongly indicates a promising role of the finite group in problems of resolutions of quotient singularities. In particular, a plausible method has been suggested on the study of geometry of orbifolds through the group representation theory. It has been known that McKay correspondence [16] between representations of Kleinian groups and affine $A-D-E$ root diagrams has revealed a profound geometrical structure on the minimal resolution of the quotient surface singularity (cf. [7]). A similar connection between the finite group and general quotient singularity theories would be expected. Yet, the interest of this interplay of geometry and group representations would not only aim at the research of crepant resolutions, but also at its own right, due to possible implications on understanding some certain special kinds of group representations by engaging the rich algebraic geometry techniques. 
In this article, we study problems related to the crepant resolutions of quotient singularities of higher dimension $n$ (mainly for $n \geq 4$ ). Due to the many complicated exceptional cases of the problem, we restrict ourselves here only to those of the hypersurface singularity type. The purpose of this paper is to present certain primitive results of our first attempt on the study of the higher-dimensional hypersurface orbifolds under the principle of "geometrization" of finite group representations. We give a brief account of the progress recently made. The main issue we deal with in this work is the higher-dimensional generalization of the $A$-type Kleinian surface singularity, the $A_{r}(n)$-hypersurface singularity of dimension $n$ (see (3.5) below). For $n=4$, we are able to determine the detailed structure of $A_{r}(4)$-Hilbert scheme and its relation with crepant resolutions of $\mathbb{C}^{4} / A_{r}(4)$. In the process, an explicit "flop" construction of 4folds among different crepant resolutions is found. In this article, we only sketch the main ideas behind the proof of these results, referring the reader to our forthcoming paper [2] for a more complete description of the methods and arguments used.

This paper is organized as follows. In Section 2, we give a brief introduction of the general scheme of engaging finite group representations in the birational geometry of orbifolds. Its connection with the Hilbert scheme of $G$-orbits for a finite linear group $G$ on $\mathbb{C}^{n}$, Hilb ${ }^{G}\left(\mathbb{C}^{n}\right)$, introduced in $[11,12,18]$, will be explained in Section 3 . In Section 4, we first review certain basic facts in toric geometry, which will be presented in the most suitable form for our goal, then focusing the case on $A_{r}(n)$-singularity. For $n=3$, we give a thorough discussion on the explicit toric structure of Hilb ${ }^{A_{r}(3)}\left(\mathbb{C}^{3}\right)$ as an illustration of the general result obtained by Nakamura on abelian group $G$ in [19]. For a finite group in $\mathrm{SL}_{3}(\mathbb{C})$, a recent result in [1] has shown that $\mathrm{Hilb}^{G}\left(\mathbb{C}^{3}\right)$ is a crepant resolution of $\mathbb{C}^{3} / G$. In Section 5 , we deal with a special case of 4 -dimensional orbifold with $G=A_{1}(4)$, and derive the detailed structure of $\operatorname{Hilb}^{G}\left(\mathbb{C}^{4}\right)$. Its relation with the crepant resolutions of $\mathbb{C}^{4} / G$ is given, so is the "flop" relation among crepant resolutions. In Section 6, we describe the result of $G=A_{r}(4)$ for the arbitrary $r$, then end with some concluding remarks.

NotATiOns. To present our work, we prepare some notations. By an orbifold we always mean the orbit space for a finite group action on a smooth complex manifold. For a finite group $G$, we denote

$$
\operatorname{Irr}(G)=\left\{\rho: G \rightarrow \operatorname{GL}\left(V_{\rho}\right) \text { an irreducible representative of } G\right\} .
$$

The trivial representation of $G$ will be denoted by 1 . For a $G$-module $W$, that is, a $G$-linear representation on a vector space $W$, one has the canonical irreducible decomposition

$$
W=\bigoplus_{\rho \in \operatorname{Irr}(G)} W_{\rho}
$$

where $W_{\rho}$ is a $G$-submodule of $W, G$-isomorphic to $V_{\rho} \otimes W_{\rho}^{0}$ for some trivial $G$-module $W_{\rho}^{0}$. The vector space $W_{\rho}$ will be called the $\rho$-factor of the $G$-module $W$.

For an analytic variety $X$, we do not distinct the notions of vector bundle and locally free $O_{X}$-sheaf over $X$. For a vector bundle $V$ over $X$, an automorphisms of $V$ means a linear automorphism with the identity on $X$. If the bundle $V$ is acted on by a group $G$ as bundle automorphisms, we call $V$ a $G$-bundle. 
2. Representation theory in algebraic geometry of orbifolds. In this paper, $G$ always denotes a finite (nontrivial) subgroup of $\mathrm{GL}_{n}(\mathbb{C})$ for $n \geq 2$, and $S_{G}:=\mathbb{C}^{n} / G$ with the canonical projection,

$$
\pi_{G}: \mathbb{C}^{n} \longrightarrow S_{G}
$$

and $o:=\pi_{G}(0) \in S_{G}$. When $G$ is a subgroup of $\operatorname{SL}_{n}(\mathbb{C})$, which will be our main concern later in this paper, $G$ acts on $\mathbb{C}^{n}$ freely outside a finite collection of linear subspaces with codimension greater than or equal to 2 . Then the orbifold $S_{G}$ has a nonempty singular set, $\operatorname{Sing}\left(S_{G}\right)$, of codimension greater than or equal to 2, in fact, $o \in \operatorname{Sing}\left(S_{G}\right)$.

For $G$ in $\mathrm{GL}_{n}(\mathbb{C}), S_{G}$ is a singular variety in general. By a birational morphism of a variety over $S_{G}$, we always mean a proper birational morphism $\sigma$ from variety $X$ to $S_{G}$ which defines a biregular map between $X \backslash \sigma^{-1}\left(\operatorname{Sing}\left(S_{G}\right)\right)$ and $S_{G} \backslash \operatorname{Sing}\left(S_{G}\right)$,

$$
\sigma: X \rightarrow S_{G}
$$

One has the commutative diagram

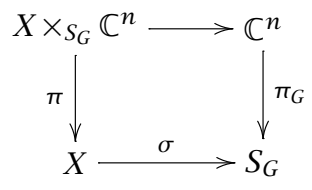

Denote by $\mathscr{F}_{X}$ the coherent $\mathscr{O}_{X}$-sheaf over $X$ obtained by the push-forward of the structure sheaf of $X \times{ }_{S_{G}} \mathbb{C}^{n}$,

$$
\mathscr{F}_{X}:=\pi_{*} \mathrm{O}_{X \times S_{G}} \mathbb{C} .
$$

The sheaf $\mathscr{F}_{X}$ has the following functorial property, namely, for $X, X^{\prime}$ birational over $S_{G}$ with the commutative diagram,

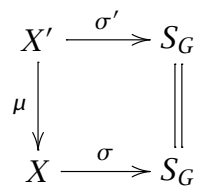

one has a canonical morphism, $\mu^{*} \mathscr{F}_{X} \rightarrow \mathscr{F}_{X^{\prime}}$. In particular, with the morphism (2.2) we have the $\mathrm{O}_{X}$-morphism

$$
\sigma^{*}\left(\pi_{G *} \mathbb{O}_{\mathbb{C}^{n}}\right) \longrightarrow \mathscr{F}_{X}
$$

Furthermore, all the morphisms in (2.3) and (2.5) are compatible with the natural $G$ structure on $\mathscr{F}_{X}$ induced from the $G$-action on $\mathbb{C}^{n}$ via (2.3). One has the canonical $G$-decomposition of $\mathscr{F}_{X}$

$$
\mathscr{F}_{X}=\bigoplus_{\rho \in \operatorname{Irr}(G)}\left(\mathscr{F}_{X}\right)_{\rho}
$$

where $\left(\mathscr{F}_{X}\right)_{\rho}$ is the $\rho$-factor of $\mathscr{F}_{X}$, and it is a coherent $\mathcal{O}_{X}$-sheaf over $X$. The geometrical 
fiber of $\mathscr{F}_{X},\left(\mathscr{F}_{X}\right)_{\rho}$ over an element $x$ of $X$ are defined by

$$
\mathscr{F}_{X, x}=k(x) \bigotimes_{\mathscr{O}_{X}} \mathscr{F}_{X}, \quad\left(\mathscr{F}_{X}\right)_{\rho, x}=k(x) \bigotimes_{\mathscr{O}_{X}}\left(\mathscr{F}_{X}\right)_{\rho},
$$

where $k(x)\left(:=\mathcal{O}_{X, x} / M_{x}\right)$ is the residue field at $x$. Over $X-\sigma^{-1}\left(\operatorname{Sing}\left(S_{G}\right)\right), \mathscr{F}_{X}$ is a vector bundle of rank $|G|$ with the regular $G$-representation on each geometric fiber. Hence $\left(\mathscr{F}_{X}\right)_{\rho}$ is a vector bundle over $X-\sigma^{-1}\left(\operatorname{Sing}\left(S_{G}\right)\right)$ of rank equal to $\operatorname{dim} V_{\rho}$. For $x \in X$, there exists a $G$-invariant ideal $I(x)$ in $\mathbb{C}[Z]\left(:=\mathbb{C}\left[Z_{1}, \ldots, Z_{n}\right]\right)$ such that the following relation holds:

$$
\mathscr{F}_{X, x}=k(x) \bigotimes_{{ }_{S_{G}}} \mathscr{O}_{\mathbb{C} n}(x) \simeq \frac{\mathbb{C}[Z]}{I(x)} .
$$

We have $\left(\mathscr{F}_{X}\right)_{\rho, x} \simeq(\mathbb{C}[Z] / I(x))_{\rho}$. The vector spaces $\mathbb{C}[Z] / I(x)$ form a family of finitedimensional $G$-modules parametrized by $x \in X$, which are equivalent to the regular representation for elements outside $\sigma^{-1}\left(\operatorname{Sing}\left(S_{G}\right)\right)$.

Set $X=S_{G}$ in (2.9). For $s \in S_{G}$, there is a $G$-invariant ideal $I(s)$ of $\mathbb{C}[Z]$; in fact, $I(s)$ is the ideal in $\mathbb{C}[Z]$ generated by the $G$-invariant polynomials vanishing at $\sigma^{-1}(s)$. Let $\tilde{I}(s)$ be the ideal of $\mathbb{C}[Z]$ consisting of all polynomials in $\mathbb{C}[Z]$ vanishing at $\sigma^{-1}(s)$. Then $\widetilde{I}(s)$ is a $G$-invariant ideal, and we have

$$
\widetilde{I}(s) \supset I(s) .
$$

In particular, for $s=o$, we have

$$
\tilde{I}(o)=\mathbb{C}[Z]_{0}, \quad I(o)=\mathbb{C}[Z]_{0}^{G} \mathbb{C}[Z],
$$

where the subscript 0 means the maximal ideal with polynomials vanishing at the origin. For a birational variety $X$ over $S_{G}$ via $\sigma$ in (2.2), the following relations of $G$ invariant ideals of $\mathbb{C}[Z]$ hold:

$$
\widetilde{I}(s) \supset I(x) \supset I(s), \quad x \in X, s=\sigma(x) .
$$

A certain connection exists between algebraic geometry and $G$-modules through the variety $X$. For $x \in X$, there is a direct sum $G$-decomposition of $\mathbb{C}[Z]$,

$$
\mathbb{C}[Z]=I(x)^{\perp} \oplus I(x) .
$$

Here $I(x)^{\perp}$ is a finite-dimensional subspace of $\mathbb{C}[Z]$ which is $G$-isomorphic to $\mathbb{C}[Z] / I(x)$. Similarly, we have the $G$-decomposition of $\mathbb{C}[Z]$ for $s=\sigma(x) \in S_{G}$,

$$
\mathbb{C}[Z]=I(s)^{\perp} \oplus I(s), \quad \mathbb{C}[Z]=\tilde{I}(s)^{\perp} \oplus \tilde{I}(s),
$$

such that the following relations hold for the finite-dimensional $G$-modules:

$$
\widetilde{I}(s)^{\perp} \subset I(x)^{\perp} \subset I(s)^{\perp} .
$$

Consider the canonical $G$-decomposition of $I(x)^{\perp}$,

$$
I(x)^{\perp}=\bigoplus_{\rho \in \operatorname{Irr}(G)} I(x)_{\rho}^{\perp} .
$$


Note that $I(x) \stackrel{\perp}{\rho}$ is isomorphic to a positive finite copies of $V_{\rho}$. Then the affine structure of $X$ near $x$ is determined by the $\mathbb{C}$-algebra generated by all the $G$-invariant rational functions $f(Z)$ such that $f(Z) I(x) \stackrel{\perp}{\rho} \subset I(x)$ for some $\rho$.

3. Hilbert scheme of finite group orbits. Among the varieties $X$ that are birational over $S_{G}$ with $\mathscr{F}_{X}$ a vector bundle, there exists a minimal object, called the $G$-Hilbert scheme in $[11,12,18]$,

$$
\sigma_{\text {Hilb }}: \operatorname{Hilb}^{G}\left(\mathbb{C}^{n}\right) \longrightarrow S_{G}
$$

For another $X$, the map (2.2) can be factored through a birational morphism $\lambda$ from $X$ onto $\operatorname{Hilb}^{G}\left(\mathbb{C}^{n}\right)$ via $\sigma_{\text {Hilb }}$,

$$
\lambda: X \rightarrow \operatorname{Hilb}^{G}\left(\mathbb{C}^{n}\right) .
$$

In fact, the ideal $I(x), x \in X$, of (2.9) are with the co-length $|G|$, which gives rise to the above map $\lambda$ of $X$ to $\operatorname{Hilb}^{G}\left(\mathbb{C}^{n}\right)$. We denote $X_{G}$ as the normal variety over $S_{G}$ defined by

$$
X_{G}:=\text { normalization of } \operatorname{Hilb}^{G}\left(\mathbb{C}^{n}\right), \quad \sigma_{G}: X_{G} \longrightarrow S_{G} .
$$

By the fact that every biregular automorphism of $S_{G}$ can always be lifted to one on $\operatorname{Hilb}^{G}\left(\mathbb{C}^{n}\right)$, hence on $X_{G}$, one has the following result.

LEMMA 3.1. Denote by $\operatorname{Aut}\left(S_{G}\right)$ the group of biregular automorphisms of $S_{G}$. Then $\operatorname{Hilb}^{G}\left(\mathbb{C}^{n}\right)$ and $X_{G}$ are varieties over $S_{G}$ with the $\operatorname{Aut}\left(S_{G}\right)$-equivariant covering morphisms.

By the definition of $\operatorname{Hilb}^{G}\left(\mathbb{C}^{n}\right)$, an element $p$ of $\operatorname{Hilb}^{G}\left(\mathbb{C}^{n}\right)$ represents a $G$-invariant ideal $I(p)$ of $\mathbb{C}[Z]$ of co-length $|G|$. The fiber of the vector bundle $\mathscr{F}_{\mathrm{Hilb}^{G}\left(\mathbb{C}^{n}\right)}$ over $p$ can be identified with the regular $G$-representation space $\mathbb{C}[Z] / I(p)$. Our study mainly concentrates on the relation of crepant resolutions of $S_{G}$ and $\operatorname{Hilb}^{G}\left(\mathbb{C}^{n}\right)$. For this purpose we assume for the rest of the paper that the group $G$ is a subgroup of $\mathrm{SL}_{n}(\mathbb{C})$

$$
G \subset \mathrm{SL}_{n}(\mathbb{C})
$$

which is the same to say that $S_{G}$ has the Gorenstein quotient singularity. For $n=2$, these groups were classified by Klein [14] into $A-D-E$ types, the singularities are called Kleinian singularities. The minimal resolution $\hat{S}_{G}$ of $S_{G}$ has the trivial canonical bundle (i.e., crepant), by [9]. In $[11,12,18]$, Ito and Nakamura showed that $\mathrm{Hilb}^{G}\left(\mathbb{C}^{2}\right)$ is equal to the minimal resolution $\widehat{S}_{G}$. For $n=3$, it has been known that there exist crepant resolutions for a 3-dimensional Gorenstein orbifold (see [26] and the references therein). Two different crepant resolutions of the same orbifolds are connected by a sequence of flop processes (cf. [22]). It was expected that $\operatorname{Hilb}^{G}\left(\mathbb{C}^{3}\right)$ is one of those crepant resolutions. The assertion has been confirmed in the abelian group case in [19], and in general by [1].

For the motivation of our later study on the higher-dimensional singularities, we now illustrate the relation between $G$-Hilbert scheme and the minimal resolution in dimension 2, that is, surface singularities. For the rest of this section, we are going to 
describe the structure of $\operatorname{Hilb}^{G}\left(\mathbb{C}^{2}\right)$ for the $A$-type Kleinian group,

$$
G=A_{r}:=\left\{\left(\begin{array}{cc}
\epsilon & 0 \\
0 & \epsilon^{-1}
\end{array}\right) \mid \epsilon^{r+1}=1\right\}, \quad r \geq 1 .
$$

The affine ring of $\mathbb{C}^{2}$ is $\mathbb{C}[Z]\left(=\mathbb{C}\left[Z_{1}, Z_{2}\right]\right)$ and $G$-invariant polynomials is the algebra generated by

$$
Y_{1}:=Z_{1}^{r+1}, \quad Y_{2}:=Z_{2}^{r+1}, \quad X:=Z_{1} Z_{2} .
$$

Hence the ideal $I(o)$ in $\mathbb{C}[Z]$ for $o \in S_{G}$ is equal to $\left\langle Z_{1}^{r+1}, Z_{1}^{r+1}, Z_{1} Z_{2}\right\rangle$, and $Z_{1}^{k}, Z_{2}^{k}$ $(0 \leq k \leq r)$, form a basis of the $G$-module $I(o)^{\perp}$. For a nontrivial character $\rho, I(o)_{\rho}^{\perp}$ is of dimension 2, in fact, it has a basis consisting of a pair, $Z_{1}^{k}, Z_{2}^{r+1-k}$, for some $k$. With the method of continued fraction [9], it is known that the minimal resolution $\hat{S}_{G}$ of $S_{G}$ has the trivial canonical bundle with an open affine cover $\left\{u_{k}\right\}_{k=0}^{r}$, where the coordinates $\left(u_{k}, v_{k}\right)$ of $u_{k}$ is expressed by

$$
u_{k} \simeq \mathbb{C}^{2} \ni\left(u_{k}, v_{k}\right)=\left(Z_{1}^{k+1} Z_{2}^{-r+k}, Z_{1}^{-k} Z_{2}^{r+1-k}\right) .
$$

Denote by $\hat{o}_{k}$ the element in $\hat{S}_{G}$ with the coordinate $u_{k}=v_{k}=0$. The exceptional divisor in $\hat{S}_{G}$ is $E_{1}+\cdots+E_{r}$ where $E_{j}$ is a rational (-2)-curve joining $\hat{o}_{j-1}$ and $\hat{o}_{j}$. The configuration can be realized in the following tree diagram:

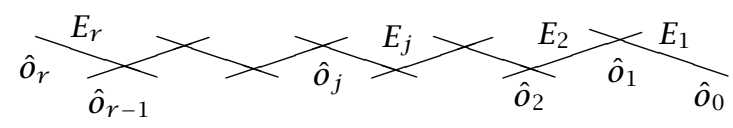

FIGURE 3.1. Exceptional curve configuration in the minimal resolution of $\mathbb{C}^{2} / A_{r}$.

It is easy to see that the ideal $I\left(\hat{o}_{k}\right)$ is given by

$$
I\left(\hat{o}_{k}\right)=\left\langle Z_{1}^{k+1}, Z_{2}^{r+1-k}, Z_{1} Z_{2}\right\rangle,
$$

hence the $G$-module $\mathbb{C}[Z] / I\left(\hat{o}_{k}\right)$ is the regular representation isomorphic to the following one,

$$
I\left(\hat{o}_{k}\right)^{\perp}=\mathbb{C}+\sum_{i=1}^{k} \mathbb{C} Z_{1}^{i}+\sum_{j=1}^{r-k} \mathbb{C} Z_{2}^{j} .
$$

One can represent monomials in (3.9) as the ones with • in Figure 3.2.

For $x \in U_{k}$, the ideal $I(x)$ has the expression

$$
I(x)=\left\langle Z_{1}^{k+1}-\alpha Z_{2}^{r-k}, Z_{2}^{r+1-k}-\beta Z_{1}^{k}, Z_{1} Z_{2}-\alpha \beta\right\rangle, \quad \alpha, \beta \in \mathbb{C} .
$$

The classes in $\mathbb{C}[Z] / I(x)$ represented by monomials in (3.9) still form a basis, hence give rise to a local frame of the vector bundle $\mathscr{F}_{\hat{S}} \hat{S}_{G}$ over $U_{k}$. The divisor $E_{k+1}$ is defined by $\beta=0$, and its element approaches to $\hat{o}_{k+1}$ as $\alpha$ tends to infinity. 


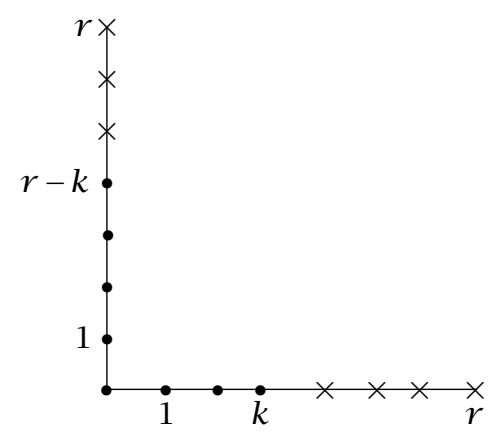

FIGURE 3.2. Representatives of $I\left(\hat{o}_{k}\right)^{\perp}\left(\simeq \mathbb{C}[Z] / I\left(\hat{o}_{k}\right)\right)$ for the minimal resolution of $\mathbb{C}^{2} / A_{r}$.

4. Abelian orbifolds and toric geometry. In this section, we discuss the abelian group case of $G$ in Section 3 using methods in toric geometry. We consider $G$ as a subgroup of the diagonal group of $\mathrm{GL}_{n}(\mathbb{C})$, denoted by $T_{0}:=\mathbb{C}^{* n}$, and regard $\mathbb{C}^{n}$ as the partial compactification of $T_{0}$,

$$
G \subset T_{0} \subset \mathbb{C}^{n}
$$

Define the $n$-torus $T$ with the toric embedding in $S_{G}\left(=\mathbb{C}^{n} / G\right)$ by

$$
T:=\frac{T_{0}}{G}, \quad T \subset S_{G} .
$$

Techniques in toric geometry rely on lattices of one-parameter subgroups, characters of $T_{0}, T$,

$$
\begin{aligned}
& N\left(:=\operatorname{Hom}\left(\mathbb{C}^{*}, T\right)\right) \supset N_{0}\left(:=\operatorname{Hom}\left(\mathbb{C}^{*}, T_{0}\right)\right), \\
& M\left(:=\operatorname{Hom}\left(T, \mathbb{C}^{*}\right)\right) \subset M_{0}\left(:=\operatorname{Hom}\left(T_{0}, \mathbb{C}^{*}\right)\right) .
\end{aligned}
$$

For our convenience, we make the following identification of $N_{0}, N$ with lattices in $\mathbb{R}^{n}$. An element $x$ in $\mathbb{R}^{n}$ has the coordinates $x_{i}$ with respect to the standard basis $\left(e^{1}, \ldots, e^{n}\right)$

$$
x=\sum_{i=1}^{n} x_{i} e^{i} \in \mathbb{R}^{n} .
$$

Then

$$
N_{0}=\mathbb{Z}^{n}\left(:=\exp ^{-1}(1)\right), \quad N=\exp ^{-1}(G),
$$

where $\exp : \mathbb{R}^{n} \rightarrow T_{0}$ is defined by $\exp (x)=\sum_{i} e^{2 \pi \sqrt{-1}} x_{i} e^{i}$. Note that $G \simeq N / N_{0}$. The dual lattice $M_{0}$ of $N_{0}$ is the standard one in the dual space $\mathbb{R}^{n *}$, and we identify it with the group of monomials of $Z_{1}, \ldots, Z_{n}$ via the correspondence

$$
I=\sum_{s=1}^{n} i^{s} e_{s} \in M_{0} \longleftrightarrow Z^{I}=\prod_{s=1}^{n} Z_{s}^{i_{s}} .
$$

The dual lattice $M$ of $N$ is the sublattice of $M_{0}$ corresponding to the set of $G$-invariant monomials. 
Over the $T$-space $S_{G}$, we now consider only those varieties $X$ which are normal and birational over $S_{G}$ with a $T$-structure, hence as it has been known, are presented by certain combinatorial data by the toric method $[4,13,20]$. Note that by Lemma 3.1, $X_{G}$ is a toric variety over $S_{G}$. In general, a toric variety over $S_{G}$ is described by a fan $\Sigma=\left\{\sigma_{\alpha} \mid \sigma \in I\right\}$ whose support equals to the first quadrant of $\mathbb{R}^{n}$, that is, a rational convex cone decomposition of the first quadrant of $\mathbb{R}^{n}$. Equivalently, it is determined by the intersection of the fan and the simplex $\triangle$ where

$$
\triangle:=\left\{x \in \mathbb{R}^{n} \mid \sum_{i} x_{i}=1, x_{j} \geq 0 \forall j\right\} .
$$

The data in $\triangle$ is given by $\Lambda=\left\{\triangle_{\alpha} \mid \alpha \in I\right\}$, where $\triangle_{\alpha}:=\sigma_{\alpha} \cap \triangle$. The $\triangle_{\alpha}$ s form a decomposition of $\triangle$ by convex subsets, having the vertices in $\triangle \cap \mathbb{Q}^{n}$. Note that for $\sigma_{\alpha}=\{\overrightarrow{0}\}$, we have $\triangle_{\alpha}=\varnothing$. We call $\Lambda$ a rational polytope decomposition of $\triangle$, and denote the corresponding toric variety by $X_{\Lambda}$. We call $\Lambda$ an integral polytope decomposition of $\triangle$ if all the vertices of $\Lambda$ are in $N$. For a rational polytope decomposition $\Lambda$ of $\triangle$, we define $\Lambda(i):=\left\{\triangle_{\alpha} \in \Lambda \mid \operatorname{dim}\left(\triangle_{\alpha}\right)=i\right\}$ for $-1 \leq i \leq n-1$, (here $\operatorname{dim}(\varnothing):=-1$ ). Then $T$-orbits in $X_{\Lambda}$ are parametrized by $\bigsqcup_{i=-1}^{n-1} \Lambda(i)$. In fact, for each $\triangle_{\alpha} \in \Lambda(i)$, there associates a $T$-orbit of the dimension $n-1-i$, denoted by orb $\left(\triangle_{\alpha}\right)$. A toric divisor in $X_{\Lambda}$ is the closure of an $(n-1)$-dimensional orbit, denoted by $D_{v}=\overline{\operatorname{orb}(v)}$ for $v \in \Lambda(0)$. The canonical sheaf of $X_{\Lambda}$ has the expression in terms of toric divisors (cf. [13]),

$$
\omega_{X_{\Lambda}}=\sigma_{X_{\Lambda}}\left(\sum_{v \in \Lambda(0)}\left(m_{v}-1\right) D_{v}\right),
$$

where $m_{v}$ is the positive integer such that $m_{v} v$ is a primitive element of $N$. In particular, the crepant property of $X_{\Lambda}$, that is, $\omega_{X_{\Lambda}}=\mathcal{O}_{X_{\Lambda}}$, is given by the integral condition of $\Lambda$. The nonsingular criterion of $X_{\Lambda}$ is the simplicial decomposition of $\Lambda$ together with the multiplicity one property, that is, for each $\Lambda_{\alpha} \in \triangle(n-1)$, the elements $m_{v} v$, $v \in \Lambda_{\alpha} \cap \Lambda(0)$, form a $\mathbb{Z}$-basis of $N$. The following results are known for toric variety over $S_{G}$ (cf. [21] and the references therein).

(1) The Euler number of $X_{\Lambda}$ is given by $\chi\left(X_{\Lambda}\right)=|\Lambda(n-1)|$.

(2) For a rational polytope decomposition $\Lambda$ of $\triangle$, any two of the following three conditions implies the third one:

$$
X_{\Lambda} \text { is nonsingular, } \quad \omega_{X_{\Lambda}}=0_{X_{\Lambda}}, \quad \chi\left(X_{\Lambda}\right)=|G| .
$$

It is easy to see that the following result holds for the sheaf $\mathscr{F}_{X_{\Lambda}}$.

LEMMA 4.1. Let $\Lambda$ be a rational polytope decomposition of $\triangle$, and let $x_{0}$ be the zerodimensional toric orbit in $X_{\Lambda}$ corresponding to an element $\triangle_{\alpha_{0}}$ in $\Lambda(n-1)$. Let $Z^{I^{(j)}}$, $1 \leq j \leq N$, be a finite collection of monomials whose classes generate the $G$-module $\mathbb{C}[Z] / I\left(x_{0}\right)$. Then the classes of $Z^{I^{(j)}}$ s also generate $\mathbb{C}[Z] / I(y)$ for $y \in \operatorname{orb}\left(\triangle_{\beta}\right)$ with $\triangle_{\beta} \subseteq \triangle_{\alpha_{0}}$.

Define

$$
A_{r}(n):=\left\{g \in \mathrm{SL}_{n}(\mathbb{C}) \mid g \text { is diagonal and } g^{r+1}=1\right\}, \quad r \geq 1 .
$$


Note that the above group for $n=2$ is the same as $A_{r}$ in (3.5). For a general $n, A_{r}(n)$ invariant polynomials in $\mathbb{C}[Z]$ are generated by the following $(n+1)$ ones:

$$
X:=\prod_{i=1}^{n} Z_{i}, \quad Y_{j}:=Z_{j}^{r+1} \quad(j=1, \ldots, n) .
$$

This implies that $S_{A_{r}(n)}$ is the singular hypersurface in $\mathbb{C}^{n+1}$,

$$
S_{A_{r}(n)}=\left\{\left(x, y_{1}, \ldots, y_{n}\right) \in \mathbb{C}^{n+1} \mid x^{r+1}=y_{1} \cdots y_{n}\right\} .
$$

For the rest of this paper, we conduct the discussion of abelian orbifolds mainly on the group $A_{r}(n)$. The ideal $I(o)$ of $\mathbb{C}[Z]$ associated to the element $o \in S_{A_{r}(n)}$ is given by

$$
I(o)=\left\langle Z_{1}^{r+1}, \ldots, Z_{n}^{r+1}, Z_{1} \cdots Z_{n}\right\rangle
$$

hence

$$
I(o)^{\perp}=\bigoplus\left\{\mathbb{C} Z^{I} \mid I=\left(i^{1}, \ldots, i^{n}\right), 0 \leq i^{j} \leq r, \prod_{j=1}^{n} i^{j}=0\right\} .
$$

For $\mathbf{1} \neq \rho \in \operatorname{Irr}\left(A_{n}(r)\right)$, the dimension of $I(o)_{\rho}^{\perp}$ is always greater than one. In fact, one can describe explicitly a set of monomial generators of $I(o)_{\rho}^{\perp}$. For example, say $I(o)_{\rho}^{\perp}$ containing an element $Z^{I}$ with $I=\left(i^{1}, \ldots, i^{n}\right), i^{1}=0$ and $i^{s} \leq i^{s+1}$, then $I(o)_{\rho}^{\perp}$ is generated by $Z^{K} s$ with $K=\left(k^{1}, \ldots, k^{n}\right)$ given by

$$
k^{s}= \begin{cases}r+1-i^{j}+i^{s} & \text { if } i^{s}<i^{j}, \\ i^{s}-i^{j} & \text { otherwise }\end{cases}
$$

here $j$ runs through 1 to $n$. In particular for $r=1$, the dimension of $I(o)_{\rho}^{\perp}$ is equal to 2 for $\rho \neq 1$, with a basis consisting of $Z^{I}, Z^{I^{\prime}}$ whose indices satisfy the relations, $0 \leq i^{s}, i^{s^{\prime}} \leq 1, i^{s}+i^{s^{\prime}}=1$ for $1 \leq s \leq n$.

For $n=3$, by the general result of Nakamura on an abelian group $G$ (see [19, Theorem 4.2$]), \operatorname{Hilb}^{A_{r}(3)}\left(\mathbb{C}^{3}\right)$ is a crepant toric variety. To illustrate this fact, we give here a direct derivation of the result by working on the explicitly described toric variety.

EXAMPLE 4.2. It is easy to see that $\triangle \cap N$ consists of the following elements:

$$
v^{m_{1}, m_{2}, m_{3}}:=\frac{1}{r+1} \sum_{j=1}^{3} m_{j} e^{j}, \quad m_{j} \in \mathbb{Z}_{\geq 0}, \sum_{j=1}^{3} m_{j}=r+1 .
$$

Denote by $\Xi$ the simplicial decomposition of $\triangle$ obtained by drawing the three lines parallel to the edges of $\triangle$ through each element of $\triangle \cap N$. The 2-simplexes in $\Xi$ (2) consists of two types of triangles, $\triangle_{u}^{m_{1}, m_{2}, m_{3}}, \triangle_{d}^{m_{1}, m_{2}, m_{3}}$ (see Figure 4.1):

$$
\begin{aligned}
& \triangle_{u}^{m_{1}, m_{2}, m_{3}}=\left\langle v^{m_{1}, m_{2}, m_{3}}, v^{m_{1}-1, m_{2}+1, m_{3}}, v^{m_{1}-1, m_{2}, m_{3}+1}\right\rangle, \\
& \triangle_{d}^{m_{1}, m_{2}, m_{3}}=\left\langle v^{m_{1}, m_{2}, m_{3}}, v^{m_{1}-1, m_{2}+1, m_{3}}, v^{m_{1}, m_{2}+1, m_{3}-1}\right\rangle,
\end{aligned}
$$

the vertices of each one form a $\mathbb{Z}$-basis of $N$. 

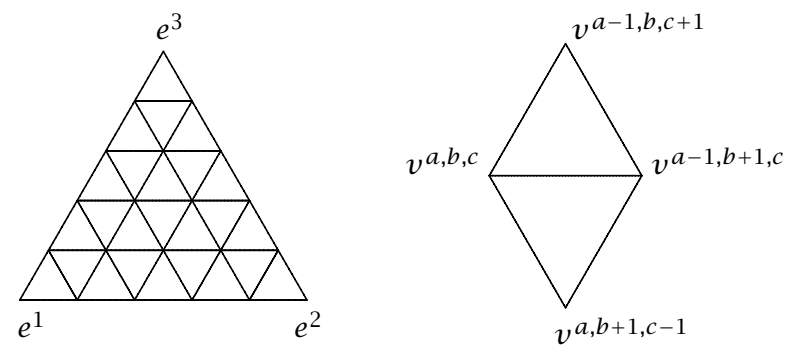

FIGURE 4.1. The right one is the simplicial decomposition $\Xi$ of $\triangle$ for $r=4$. The left one is the union of $\triangle_{\mathfrak{u}}^{a, b, c}$ and $\triangle_{d}^{a, b, c}$.

Therefore the $X_{\Xi}$ is a smooth toric variety. The 0-dimensional (toric) orbits corresponding to $\triangle_{u}^{m_{1}, m_{2}, m_{3}}, \triangle_{d}^{m_{1}, m_{2}, m_{3}}$ are denoted by $x_{u}^{m_{1}, m_{2}, m_{3}}, x_{d}^{m_{1}, m_{2}, m_{3}}$, respectively. The affine coordinate system centered at $x_{u}^{m_{1}, m_{2}, m_{3}}$ is given by

$$
U_{1}=\frac{Z_{1}^{r+2-m_{1}}}{\left(Z_{2} Z_{3}\right)^{m_{1}-1}}, \quad U_{2}=\frac{Z_{2}^{r+1-m_{2}}}{\left(Z_{1} Z_{3}\right)^{m_{2}}}, \quad U_{3}=\frac{Z_{3}^{r+1-m_{3}}}{\left(Z_{1} Z_{2}\right)^{m_{3}}} .
$$

For $y$ in $X_{\Xi}$ with the value $U_{j}$ equal to $\alpha_{j}$, by computation, the ideal $I(y)$ has the following generators:

$$
\begin{aligned}
& Z_{1}^{r+2-m_{1}}-\alpha_{1}\left(Z_{2} Z_{3}\right)^{m_{1}-1}, \quad Z_{2}^{r+1-m_{2}}-\alpha_{2}\left(Z_{3} Z_{1}\right)^{m_{2}}, \quad Z_{3}^{r+1-m_{3}}-\alpha_{3}\left(Z_{1} Z_{2}\right)^{m_{3}}, \\
& \left(Z_{1} Z_{2}\right)^{m_{3}+1}-\alpha_{1} \alpha_{2} Z_{3}^{r-m_{3}}, \quad\left(Z_{2} Z_{3}\right)^{m_{1}}-\alpha_{2} \alpha_{3} Z_{1}^{r+1-m_{1}},\left(Z_{3} Z_{1}\right)^{m_{2}+1}-\alpha_{3} \alpha_{1} Z_{2}^{r-m_{2}}, \\
& Z_{1} Z_{2} Z_{3}-\alpha_{1} \alpha_{2} \alpha_{3} .
\end{aligned}
$$

For the element $x_{u}^{m_{1}, m_{2}, m_{3}}$, that is, $\alpha_{i}=0$ for all $i$, it is easy to see that there are $(r+1)^{2}$ monomials not in $I\left(x_{u}^{m_{1}, m_{2}, m_{3}}\right)$, which form a basis of $I\left(x_{u}^{m_{1}, m_{2}, m_{3}}\right) \perp$, and give rise to a basis of $\mathbb{C}[Z] / I(y)$ for $y$ in the affine neighborhood of $x_{u}^{m_{1}, m_{2}, m_{3}}$. The same argument applies to the affine neighborhood near $x_{u}^{m_{1}, m_{2}, m_{3}}$ with the coordinate functions

$$
V_{1}=\frac{\left(Z_{2} Z_{3}\right)^{m_{1}}}{Z_{1}^{r+1-m_{1}}}, \quad V_{2}=\frac{\left(Z_{1} Z_{3}\right)^{m_{2}+1}}{Z_{2}^{r-m_{2}}}, \quad V_{3}=\frac{\left(Z_{1} Z_{2}\right)^{m_{3}}}{Z_{3}^{r+1-m_{3}}},
$$

hence the description of ideals for $y$ in $X_{\Xi}$ with $V_{j}=\beta_{j}$,

$$
\begin{aligned}
& Z_{1}^{r+2-m_{1}}-\beta_{2} \beta_{3}\left(Z_{2} Z_{3}\right)^{m_{1}-1}, \quad Z_{2}^{r+1-m_{2}}-\beta_{3} \beta_{1}\left(Z_{3} Z_{1}\right)^{m_{2}}, \quad Z_{3}^{r+2-m_{3}}-\beta_{1} \beta_{2}\left(Z_{1} Z_{2}\right)^{m_{3}-1}, \\
& \left(Z_{1} Z_{2}\right)^{m_{3}}-\beta_{3} Z_{3}^{r+1-m_{3}}, \quad\left(Z_{2} Z_{3}\right)^{m_{1}}-\beta_{1} Z_{1}^{r+1-m_{1}}, \quad\left(Z_{3} Z_{1}\right)^{m_{2}+1}-\beta_{2} Z_{2}^{r-m_{2}}, \\
& Z_{1} Z_{2} Z_{3}-\beta_{1} \beta_{2} \beta_{3} .
\end{aligned}
$$

Therefore, we have shown that $X_{\Xi}$ is birational over $\operatorname{Hilb}^{G}\left(\mathbb{C}^{3}\right)$. Now we are going to show that they are in fact the same. Let $x$ be an element in $\operatorname{Hilb}^{G}\left(\mathbb{C}^{3}\right)$ represented by a monomial ideal $J=I(x)$ (i.e., with a set of generators composed of monomials). Then the regular $G$-module $J^{\perp}$ is generated by $|G|$ monomials, and $x$ lies over the element $o$ 
of $S_{G}$, equivalently, $J$ contains the ideal $\mathbb{C}[Z]_{0}^{G}$. Denote by $l_{i}$ the smallest nonnegative integer such that $Z_{i}^{l_{i}} \in J$, by $l_{i, j}$ the smallest nonnegative integer with $\left(Z_{i} Z_{j}\right)^{l_{i, j}} \in J$ for $i \neq j$. Hence $1 \leq l_{i} \leq r+1$, and $Z_{i}^{l_{i}-1} \in J^{\perp}$, which implies $\left(Z_{1} Z_{2} Z_{3} / Z_{i}\right)^{r+2-l_{i}} \in J$. In particular, $Z_{1}^{l_{1}-1} \in J^{\perp}$ and $\left(Z_{2} Z_{3}\right)^{r+2-l_{1}} \in J$. By the description (4.15) for $I(o)^{\perp}, Z_{1}^{l_{1}}$ is the only monomial in the basis of $I(o)^{\perp}$ for the corresponding character of $G$, the same for $\left(Z_{2} Z_{3}\right)^{r+1-l_{1}}$. Hence $\left(Z_{2} Z_{3}\right)^{r+2-l_{1}} \in J^{\perp}$, which implies $l_{1}+l_{2,3}=r+2$. Similarly, we have $l_{2}+l_{1,3}=l_{3}+l_{1,2}=r+2$. Again by (4.15), $Z_{1}^{l_{1}-1} Z_{2}^{l_{1,2}-1}, Z_{2}^{r+1-l_{1}+l_{1,2}} Z_{3}^{r+2-l_{1}}$, $Z_{1}^{l_{1}-l_{1,2}} Z_{3}^{l_{3}}$ are the generators of an eigenspace in $I(o)^{\perp}$. The latter two are elements in $J$ by $\left(Z_{2} Z_{3}\right)^{r+2-l_{1}}, Z_{3}^{l_{3}} \in J$. Therefore $Z_{1}^{l_{1}-1} Z_{2}^{l_{1,2}-1} \in J^{\perp}$. Similarly, one has

$$
Z_{2}^{l_{2}-1} Z_{1}^{l_{1,2}-1}, Z_{1}^{l_{1}-1} Z_{3}^{l_{1,3}-1}, Z_{3}^{l_{3}-1} Z_{1}^{l_{1,3}-1}, Z_{2}^{l_{2}-1} Z_{3}^{l_{2,3}-1}, Z_{3}^{l_{3}-1} Z_{2}^{l_{2,3}-1} \in J^{\perp} .
$$

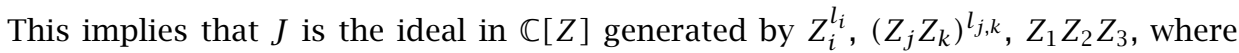
$\{i, j, k\}=\{1,2,3\}$ with $j<k$, and $l_{i}, l_{j, k}$ are integers satisfying the relations $l_{i}+l_{j, k}=$ $r+2,1 \leq l_{i} \leq r+1$. Therefore the number of monomials in $J^{\perp}$ is given by

$$
\begin{aligned}
& 1+\sum_{j=1}^{3}\left(l_{j}-1\right)+\sum_{j<k}\left(l_{j}-1\right)\left(l_{k}-1\right)-\left(l_{j}-l_{j, k}\right)\left(l_{k}-l_{j, k}\right) \\
& \quad=-\left(\sum_{j} l_{j}\right)^{2}+(4 r+7) \sum_{j} l_{j}-4(r+1)^{2}-6(r+1)-2,
\end{aligned}
$$

which is equal to $(r+1)^{2}$ by $J \in \operatorname{Hilb}^{G}\left(\mathbb{C}^{3}\right)$. The only solutions for $\sum_{j} l_{j}$ are $2(r+1)+1$, $2(r+1)+2$. Hence the ideal $J$ is characterized by integers $l_{i}$ between 1 and $r+1$ with the relation

$$
\sum_{j=1}^{3} l_{j}=2(r+1)+1, \quad \sum_{j=1}^{3} l_{j}=2(r+1)+2 .
$$

If $\sum_{j=1}^{3} l_{j}=2(r+1)+1$, the ideal $J$ corresponds to the ideal of $x_{u}^{m_{1}, m_{2}, m_{3}}$ in $X_{\Xi}$ with

$$
l_{1}=r+2-m_{1}, \quad l_{2}=r+1-m_{2}, \quad l_{3}=r+1-m_{3} .
$$

For $\sum_{j=1}^{3} l_{j}=2(r+1)+2$, the ideal $J$ corresponds to the ideal for the element $x_{d}^{m_{1}, m_{2}, m_{3}}$ in $X_{\Xi}$ with

$$
l_{1}=r+2-m_{1}, \quad l_{2}=r+1-m_{2}, \quad l_{3}=r+2-m_{3} .
$$

Now by techniques of Groebner basis for ideals in $\mathbb{C}[Z]$ (cf. [3]), given a monomial order, one has a monomial ideal $\operatorname{lt}(J)$ (generated by the leading monomial of the Groebner basis of $J$ ) such that all monic monomials outside $\operatorname{lt}(J)$ form a base of $\mathbb{C}[Z] / J$. Thus, one sees that for an element in $\operatorname{Hilb}^{G}\left(\mathbb{C}^{3}\right)$ that is a $G$-invariant ideal $J^{\prime}$ with the regular $G$-module $\mathbb{C}[Z] / J^{\prime}$, there is a basis of $\mathbb{C}[Z] / J^{\prime}$ represented by monomials in $J^{\perp}$ for some $J$ previously described. This shows that $\operatorname{Hilb}^{G}\left(\mathbb{C}^{3}\right)=X_{\Xi}$.

REMARK 4.3. As in the discussion of the case for $n=2$ in Section 3, one can represent the monomial basis elements of $I\left(x_{u}^{m_{1}, m_{2}, m_{3}}\right)^{\perp}, I\left(x_{d}^{m_{1}, m_{2}, m_{3}}\right)^{\perp}$ in a pictorial way. For example, the ones in Figure 4.2 are for $r=4$, and $m_{1}=2, m_{2}=1, m_{3}=2$. 

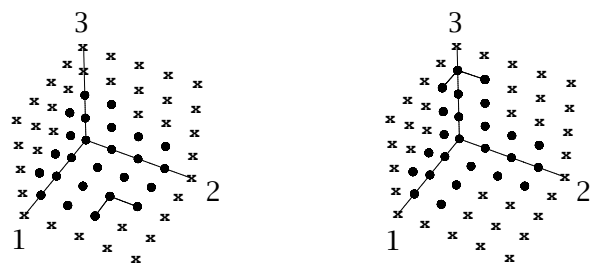

FIGURE 4.2. Graph representation of $I\left(x_{u}^{m_{1}, m_{2}, m_{3}}\right)^{\perp}, I\left(x_{d}^{m_{1}, m_{2}, m_{3}}\right)^{\perp}$ for $r=$ $4,\left(m_{1}, m_{2}, m_{3}\right)=(2,1,2)$. A dot point $\bullet$ indicates a monomial in $I^{\perp}$ while an " $\mathrm{x}$ " means one in $I$. The difference between two graphs are marked by broken segments.

5. $A_{1}(4)$-singularity and flop of 4 -folds. We now study the $A_{r}(n)$-singularity with $n \geq 4$. For simplicity, we consider the case $r=1$, that is, $G=A_{1}(n)$ (indeed, no conceptual difficulties arise for higher values of $r$ ). The $N$-integral elements in $\triangle$ are as follows:

$$
\triangle \bigcap N=\left\{e^{j} \mid 1 \leq j \leq n\right\} \bigcup\left\{v^{i, j} \mid 1 \leq i<j \leq n\right\},
$$

where $v^{i, j}:=(1 / 2)\left(e^{i}+e^{j}\right)$ for $i \neq j$. Other than the whole simplex $\triangle$, there is only one integral polytope decomposition of $\triangle$ invariant under permutations of coordinates, denoted by $\Xi$, which we now describe as follows. There are $n+1$ elements in $\Xi(n-1)$ such that $\triangle_{i}, 1 \leq i \leq n$, together with $\diamond$, where $\triangle_{i}$ is the simplex generated by $e^{i}$ and $v^{i, j}$ for $j \neq i$, and $\diamond=$ the closure of $\triangle \backslash \bigcup_{i=1}^{n} \triangle_{i}$. In fact, $\diamond$ is the convex hull spanned by all the $v^{i, j}$ for $i \neq j$. The lower-dimensional polytopes of $\Xi$ are given by the faces of those in $\Xi(n-1)$. Then $X_{\Xi}$ has the trivial canonical sheaf. However, only for $n=2,3$, $X_{\Xi}$ is a crepant resolution of $S_{A_{1}(n)}$ (cf. [21]). For $n=4$, one has the following result.

LEMMA 5.1. For $n=4$, the toric variety $X_{\Xi}$ is smooth except one isolated singularity, which is the 0-dimensional T-orbit corresponding to $\diamond$.

Proof. In general, for $n \geq 4$, it is easy to see that for each $i$, the vertices of $\triangle_{i}$ form a $\mathbb{Z}$-basis of $N$, for example, say $i=1$, it follows from $\left|A_{1}(n)\right|=2^{n-1}$, and

$$
\operatorname{det}\left(e^{1}, v^{1,2}, \ldots, v^{1, n}\right)=\frac{1}{2^{n-1}} .
$$

Hence $X_{\Xi}$ is nonsingular near the $T$-orbits associated to simplices in $\triangle_{i}$. As $\diamond$ is not a simplex, orb $(\diamond)$ is always a singular point of $X_{\Xi}$. For $n=4$, the statement of smoothness of $X_{\Xi}$ except orb $(\diamond)$ follows from the fact that for $1 \leq i \leq 4$, the vertices $v^{i, j}$ $(j \neq i)$ of $X_{\Xi}$, together with $(1 / 2) \sum_{j=1}^{4} e^{j}$, form a $N$-basis.

REMARK 5.2. For $n \geq 4$, the following properties hold for 0 -dimensional $T$-orbits of $X_{\Xi}$.

(1) Let $x_{j}:=\operatorname{orb}\left(\triangle_{j}\right) \in X_{\Xi}$ for $1 \leq j \leq n$. The inverse of the matrix of vertices of $\triangle_{j}$,

$$
\left(v^{1, j}, \ldots, v^{j-1, j}, e^{j}, v^{j+1, j}, \ldots, v^{n, j}\right)^{-1},
$$


gives rise to affine coordinates $\left(U_{1}, \ldots, U_{n}\right)$ around $x_{j}$

$$
U_{i}=Z_{i}^{2} \quad(i \neq j), \quad U_{j}=\frac{Z_{j}}{Z_{1} \cdots \check{Z}_{j} \cdots Z_{n}} .
$$

Hence

$$
I\left(x_{j}\right)=\left\langle Z_{j}, Z_{i}^{2}, i \neq j\right\rangle+I_{A_{1}(n)}
$$

with the regular $A_{1}(n)$-module isomorphism,

$$
\frac{\mathbb{C}[Z]}{I\left(x_{j}\right)} \simeq \bigoplus\left\{\mathbb{C} Z^{I} \mid I=\left(i_{1}, \ldots, i_{n}\right), i_{j}=0, i_{k}=0,1 \text { for } k \neq j\right\}
$$

(2) We denote by $x_{\diamond}$ the element orb $(\diamond)$ in $X_{\Xi}, x_{\diamond}:=\operatorname{orb}(\diamond)$. The singular structure of $x_{\diamond}$ is determined by those $A_{1}(n)$-invariant polynomials, corresponding to $M$ integral elements in the cone dual to the one generated by $\diamond$ in $N_{\mathbb{R}}$. It is easy to see that these polynomials are generated by the following ones:

$$
X_{j}:=Z_{j}^{2}, \quad Y_{j}:=\frac{Z_{1} \cdots \check{Z}_{j} \cdots Z_{n}}{Z_{j}} .
$$

Hence we have

$$
I\left(x_{\diamond}\right)=\left\langle Z_{1} \cdots \check{Z}_{j} \cdots Z_{n}\right\rangle_{1 \leq j \leq n}+I_{A_{1}(n)} .
$$

Note that for $n=3, Y_{j}$ 's form the minimal generators for the invariant polynomials, which implies the smoothness of $X_{\Xi}$. For $n \geq 4, x_{\diamond}$ is an isolated singularity, but not of the hypersurface type. For $n=4$, the $X_{j}, Y_{j}(1 \leq j \leq 4)$ form a minimal set of generators of invariant polynomials, hence the structure near $x_{\diamond}$ in $X_{\Xi}$ is the 4dimensional affine variety in $\mathbb{C}^{8}$ defined by the relations

$$
\left(x_{i}, y_{i}\right)_{1 \leq i \leq 4} \in \mathbb{C}^{8}, \quad x_{i} y_{i}=x_{j} y_{j}, \quad x_{i} x_{j}=y_{i^{\prime}} y_{j^{\prime}},
$$

where $i \neq j$ and $\left\{i^{\prime}, j^{\prime}\right\}$ is the complementary pair of $\{i, j\}$.

For the rest of this section, we consider only the case $n=4$. We discuss the crepant resolutions of $S_{A_{1}(4)}$, and its relation with $\operatorname{Hilb}^{A_{1}(4)}\left(\mathbb{C}^{4}\right)$. Now the simplex $\triangle$ is a tetrahedron, and $\diamond$ is an octahedron, on which the symmetric group $\mathfrak{G}_{4}$ acts as the standard representation. The dual polygon of $\diamond$ is the cubic. Faces of the octahedron $\diamond$ are labelled by $F_{j}, F_{j}^{\prime}$ for $1 \leq j \leq 4$, where

$$
F_{j}=\diamond \cap \triangle_{j}, \quad F_{j}^{\prime}=\left\{\sum_{i=1}^{4} x_{j} e^{j} \in \diamond \mid x_{j}=0\right\} .
$$

The dual of $F_{j}, F_{j}^{\prime}$ in the cubic are vertices, denoted by $j, j^{\prime}$ as in Figure 5.1

Consider the rational simplicial decomposition $\Xi^{*}$ of $\triangle$, which is a refinement of $\Xi$ by adding the center

$$
c:=\frac{1}{4} \sum_{j=1}^{4} e^{j}
$$

as a vertex with the barycentric decomposition of $\diamond$ in $\Xi$. Note that $c \notin N$ and $2 c \in N$. See Figure 5.2. 

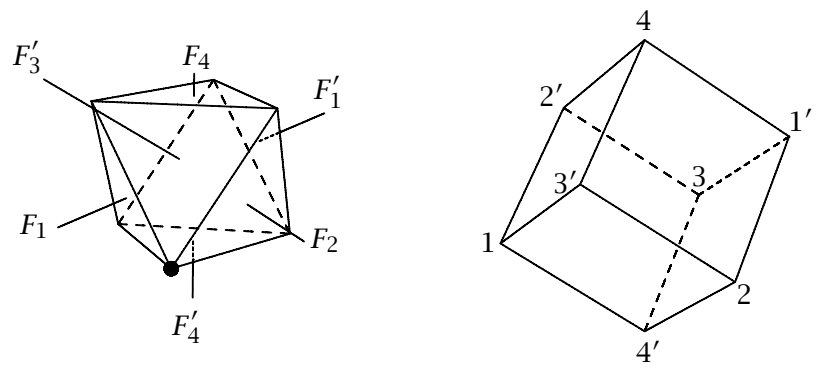

FIGURE 5.1. Dual pair of octahedron and cube. Faces $F_{j}, F_{j}^{\prime}$ of the octahedron on the left dual to vertices $j, j^{\prime}$ of the cube on the right. The face of the cube with vertices $1,4^{\prime}, 2,3^{\prime}$ corresponds to the dot "•" in the octahedron.

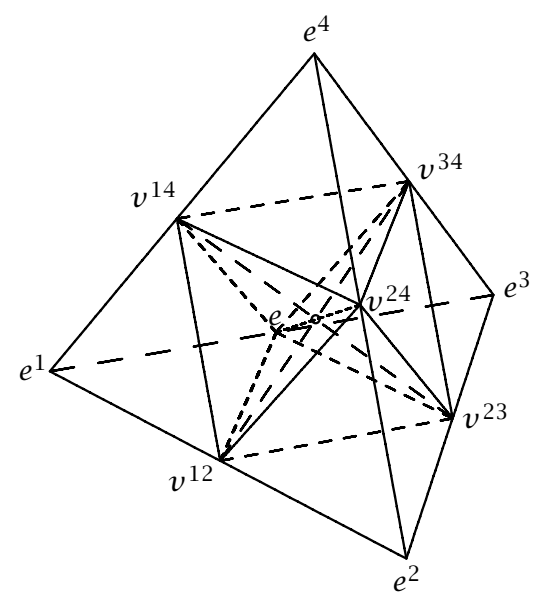

FIGURE 5.2. The rational simplicial decomposition $\Xi^{*}$ of $\triangle$ for $n=4, r=1$.

THEOREM 5.3. For $G=A_{1}(4)$, there is

$$
\operatorname{Hilb}^{A_{1}(4)}\left(\mathbb{C}^{4}\right)=X_{A_{1}(4)} \simeq X_{\Xi *},
$$

which is nonsingular with the canonical bundle $\omega=O_{X_{\Xi *}}(E)$, where $E$ is an irreducible divisor isomorphic to the triple product of $\mathbb{P}^{1}$,

$$
E=\mathbb{P}^{1} \times \mathbb{P}^{1} \times \mathbb{P}^{1} .
$$

Furthermore for $\{i, j, k\}=\{1,2,3\}$, the normal bundle of $E$ when restricted on the $\mathbb{P}^{1}$. fiber, $\mathbb{P}_{k}^{1}$, for the projection on $\mathbb{P}^{1} \times \mathbb{P}^{1}$ via the $(i, j)$ th factor,

$$
p_{k}: E \rightarrow \mathbb{P}^{1} \times \mathbb{P}^{1}
$$

is the (-1)-hyperplane bundle

$$
\mathcal{O}_{X_{\Xi *}}(E) \otimes \mathcal{O}_{\mathbb{P}_{k}^{1}} \simeq \mathcal{O}_{\mathbb{P} 1}(-1) .
$$

Proof. By Lemma 5.1 and Remark 5.2(1) after that, one can see the smoothness of $X_{\Xi *}$ on the affine chart corresponding to $\triangle_{j}$, also its relation with $\operatorname{Hilb}^{G}\left(\mathbb{C}^{4}\right)$. For 
the rest of simplexes, the octahedron $\diamond$ of $\Xi$ is decomposed into eight simplexes corresponding to the faces $F_{j}, F_{j}^{\prime}$ of $\diamond$. Denote by $C_{j}\left(C_{j}^{\prime}\right)$ the simplex of $\Xi^{*}$ spanned by $c$ and $F_{j}$ (resp., $F_{j}^{\prime}$ ), and $x_{C_{j}}, x_{C_{j}^{\prime}}$ the elements in $X_{\Xi *}$ of the corresponding $T$-orbit. First we show that for $x=x_{C_{j}}, x_{C_{j}^{\prime}}$, $\mathscr{F}_{\Xi^{*}, x}$ is a regular $G$-module. It is easy to see that the vertices of $F_{j}$ together with $2 c$ form an integral basis of $N$, the same for the vertices of $F_{j}^{\prime}$. For the convenience of notation, we can set $j=1$, without loss of generality. Then we have the integral basis of $M$ for the cones, dual to $C_{1}, C_{1}^{\prime}$ as follows:

$$
\begin{aligned}
& \text { cone }\left(C_{1}\right)^{*}:\left(2 c, v^{1,2}, v^{1,3}, v^{1,4}\right)^{-1}=\left(\begin{array}{cccc}
-1 & 1 & 1 & 1 \\
1 & 1 & -1 & -1 \\
1 & -1 & 1 & -1 \\
1 & -1 & -1 & 1
\end{array}\right) \text {, } \\
& \text { cone }\left(C_{1}^{\prime}\right)^{*}:\left(2 c, v^{2,3}, v^{2,4}, v^{3,4}\right)^{-1}=\left(\begin{array}{cccc}
2 & 0 & 0 & 0 \\
-1 & 1 & 1 & -1 \\
-1 & 1 & -1 & 1 \\
-1 & -1 & 1 & 1
\end{array}\right) .
\end{aligned}
$$

Therefore, the following 4 functions form a smooth coordinate of $X_{\Xi *}$ near $x_{C_{j}}$ for $j=1$,

$$
U_{1}=\frac{Z_{2} Z_{3} Z_{4}}{Z_{1}}, \quad U_{2}=\frac{Z_{1} Z_{2}}{Z_{3} Z_{4}}, \quad U_{3}=\frac{Z_{1} Z_{3}}{Z_{2} Z_{4}}, \quad U_{4}=\frac{Z_{1} Z_{4}}{Z_{2} Z_{3}}
$$

and one has

$$
I\left(x_{C_{1}}\right)=\left\langle Z_{2} Z_{3} Z_{4}, Z_{1} Z_{2}, Z_{1} Z_{3}, Z_{1} Z_{4}\right\rangle+I_{G}
$$

Similarly, the coordinates near $x_{C_{j}^{\prime}}$ for $j=1$ are given by

$$
U_{1}^{\prime}=Z_{1}^{2}, \quad U_{2}^{\prime}=\frac{Z_{2} Z_{3}}{Z_{1} Z_{4}}, \quad U_{3}^{\prime}=\frac{Z_{2} Z_{4}}{Z_{1} Z_{3}}, \quad U_{4}^{\prime}=\frac{Z_{3} Z_{4}}{Z_{1} Z_{2}}
$$

and we have

$$
I\left(x_{C_{1}^{\prime}}\right)=\left\langle Z_{2} Z_{3}, Z_{2} Z_{4}, Z_{3} Z_{4}\right\rangle+I_{G}
$$

It is easy to see that the $G$-modules, $\mathbb{C}[Z] / I\left(x_{C_{1}}\right), \mathbb{C}[Z] / I\left(x_{C_{1}}\right)$, are both equivalent to the regular representation. Therefore, the ideals $I(x)$ for $x=x_{\triangle_{j}}, x_{C_{j}}, x_{C_{j}^{\prime}}(1 \leq$ $j \leq 4)$, give rise to distinct elements in $\operatorname{Hilb}^{A_{1}(4)}\left(\mathbb{C}^{4}\right)$. In fact, one can show that $X_{\Xi *}=$ $\operatorname{Hilb}^{A_{1}(4)}\left(\mathbb{C}^{4}\right)$ (for the details, see [2]). By (4.8), the canonical bundle of $X_{\Xi *}$ is given by

$$
\omega_{X_{\Xi *}}=O_{X_{\Xi *}}(E),
$$

where $E$ is the toric divisor $D_{c}$. It is known that $E$ is a 3-dimensional complete toric variety arised from the star of $c$ in $\Xi^{*}$, which is given by the octahedron in Figure 5.1; in fact, the cube in Figure 5.1 represents the toric orbits' structure. Therefore, $E$ is isomorphic to the triple product of $\mathbb{P}^{1}$ as in (5.13). The conclusion of the normal 


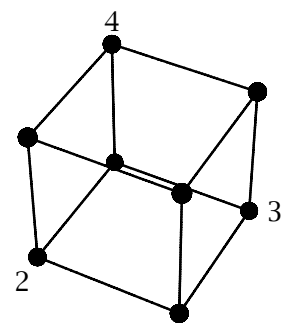

FIGURE 5.3. The monomial basis of the $G$-module $I\left(x_{\triangle_{1}}\right)^{\perp}\left(\simeq \mathbb{C}[Z] / I\left(x_{\triangle_{1}}\right)\right)$ in the $Z_{2}-Z_{3}-Z_{4}$ space.
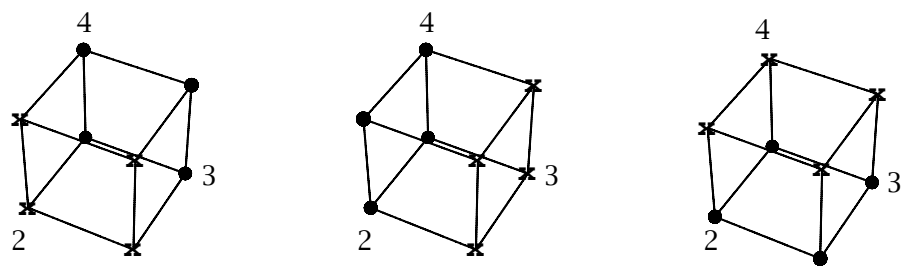

FIGURE 5.4. The corresponding $I^{\perp}$-graph for the simplex $\Delta_{2}, \Delta_{3}$, and $\Delta_{4}$ in $X_{\Xi} * \mathrm{~S}$. A dot point $\bullet$ means a monomial in $I\left(x_{\triangle_{i}}\right)^{\perp}$, while an "x" means one in $I\left(x_{\triangle_{i}}\right)$.

bundle of $E$ restricting on each $\mathbb{P}^{1}$-fiber will follow from techniques in toric geometry. For example, for fibers over the projection of $E$ onto the $\left(\mathbb{P}^{1}\right)^{2}$ corresponding to the 2-convex set spanned by $v^{1,2}, v^{1,3}, v^{3,4}, v^{2,4}$, one can perform the computation as follows. Let $\left(U_{1}, U_{2}, U_{3}, U_{4}\right)$ be the local coordinates near $x_{C_{4}^{\prime}}$ dual to the $N$-basis $\left(2 c, v^{1,2}, v^{1,3}, v^{2,3}\right)$, similarly the local coordinate $\left(W_{1}, W_{2}, W_{3}, W_{4}\right)$ near $x_{C_{1}}$ dual to $\left(2 c, v^{1,2}, v^{1,3}, v^{1,4}\right)$. By $2 c=v^{1,4}+v^{2,3}$, one has the relations

$$
U_{1}=W_{1} W_{4}, \quad U_{4}=W_{4}^{-1}, \quad U_{j}=W_{j}(j=2,3) .
$$

This shows that the restriction of the normal bundle of $E$ on a fiber $\mathbb{P}^{1}$ over $\left(U_{2}, U_{3}\right)$ plane is the $(-1)$-hyperplane bundle.

The sheaf $\mathscr{F}_{X_{\Xi}}$ for $X_{\Xi *}$ in Theorem 5.3 is a vector bundle with the regular $G$-module on each fiber. The local frame of the vector bundle is provided by the structure of $\mathbb{C}[Z] / I(x)$ for $x$ being the zero-dimensional toric orbit of $X_{\Xi^{*}}$. One can have a pictorial realization of monomial basis of these $G$-representations as follows. We start with the element $x_{\triangle_{1}}$, and the identification, $\mathbb{C}[Z] / I\left(x_{\triangle_{1}}\right)=I\left(x_{\triangle_{1}}\right)^{\perp}$. The eigenbasis of the $G$-module $I\left(x_{\triangle_{1}}\right)^{\perp}$ is given by monomials in the diagram of Figure 5.3.

By the fact that the $\rho$-eigenspace of $I(o)^{\perp}$ for the element $o \in S_{G}$ has the dimension 2 for a nontrivial character $\rho$, one can present the data of the regular $G$ module $\mathbb{C}[Z] / I(x)$ for another element $x$ by indicating the ones to be replaced in the Figure 5.3, which will be marked by $\mathbf{x}$. All of the replacement are listed in Figures 5.4, 5.5, and 5.6. 

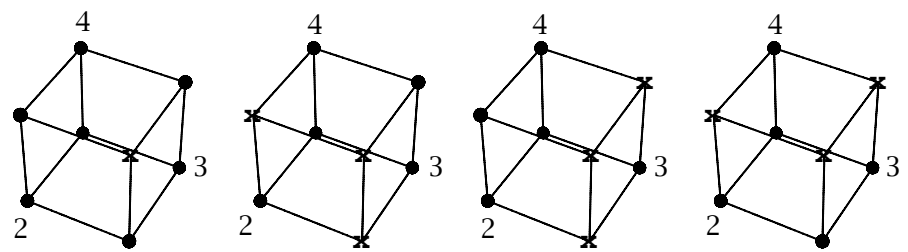

FiguRE 5.5. The corresponding $I^{\perp}$-graph for the simplex $C_{1}, C_{2}, C_{3}$, and $C_{4}$.
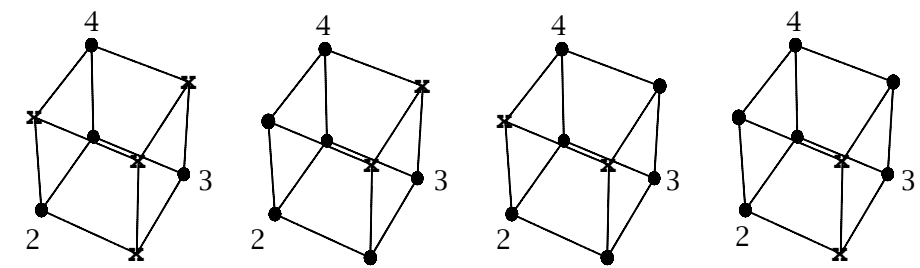

FIGURE 5.6. The corresponding $I^{\perp}$-graph for the simplex $C_{1}, C_{2}, C_{3}$, and $C_{4}$.

By the standard blowing-down criterion of an exceptional divisor, the property (5.15), ensures the existence of a smooth 4 -fold $\left(X_{\Xi *}\right)_{k}$ by blowing down the family of $\mathbb{P}^{1} \mathrm{~s}$ along the projection $p_{k}$ (5.14) for each $k$. In fact, $\left(X_{\Xi *}\right)_{k}$ is also a toric variety $X_{\Xi_{k}}$ where $\Xi_{k}$ is the refinement of $\Xi$ by adding the segment connecting $v^{k, 4}$ and $v^{i, j}$ to divide the central polygon $\diamond$ into 4 simplexes, where $\{i, j, k\}=\{1,2,3\}$. Each $X_{\Xi_{k}}$ is a crepant resolution of $X_{\Xi}\left(=S_{A_{1}(4)}\right)$. We have the relation of refinements: $\Xi \prec \Xi_{k} \prec \Xi^{*}$ for $k=1,2,3$. The polyhedral decomposition in the central part $\diamond$ appeared in the refinement relation are denoted by

$$
\diamond \prec \diamond_{k} \prec \diamond^{*}, \quad k=1,2,3,
$$

of which the pictorial realization is given in Figure 5.7. The connection of smooth 4folds for different $\diamond_{k}$ can be regarded as a "flop" of 4 -folds suggested by the similar procedure in the theory of 3-dimensional birational geometry. Each one is a "small" resolution of a 4-dimensional isolated singularity defined by (5.9). Here the smallness for a resolution means one with the exceptional locus of codimension greater than or equal to 2. Hence we have shown the following result.

THEOREM 5.4. For $G=A_{1}(4)$, there are crepant resolutions of $S_{G}$ obtained by blowing down the divisor $E$ of $\operatorname{Hilb}^{G}\left(\mathbb{C}^{4}\right)$ along (5.14) in Theorem 5.3. Any two such resolutions differ by a "flop" procedure of 4 -folds.

6. $A_{r}(4)$-singularity and conclusion remarks. For $G=A_{r}(n), n \geq 4$, the structure of $\operatorname{Hilb}^{G}\left(\mathbb{C}^{n}\right)$ and its relation with possible crepant resolutions of $S_{G}$ has been an ongoing program under investigation. We have discussed the simplest case $A_{1}(4)$ in Theorem 5.3. A similar conclusion holds also for $n=4$, but an arbitrary $r$, whose proof relies on more complicated techniques. The details will be given in [2]. 

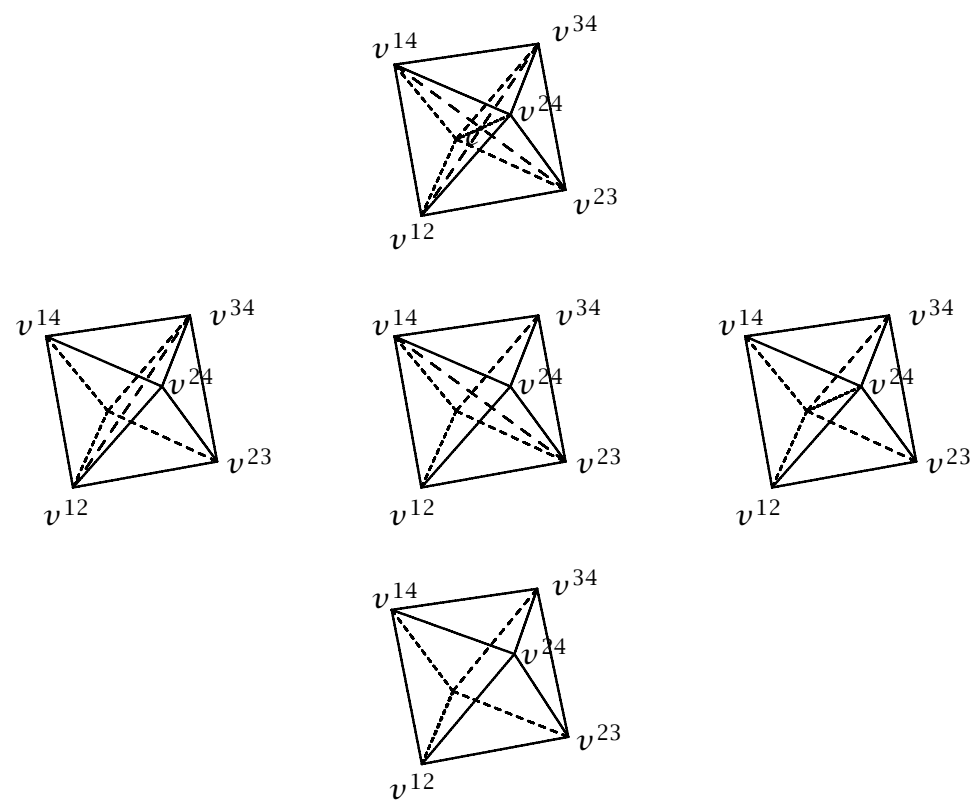

FIGURE 5.7. Toric representation of 4-dimensional flops over a common singular base and dominated by the same 4-fold.

THEOREM 6.1. The G-Hilbert scheme $\operatorname{Hilb}^{A_{r}(4)}\left(\mathbb{C}^{4}\right)$ is the nonsingular toric variety $X_{A_{r}(4)}$ with the canonical bundle

$$
\omega_{X_{A_{r}(4)}}=\mathcal{O}\left(\sum_{k=1}^{m} E_{k}\right), \quad m=\frac{r(r+1)(r+2)}{6},
$$

where $E_{k}$ 's are disjoint smooth exceptional divisors in $X_{A_{r}(4)}$, each of which satisfies conditions (5.13) and (5.15). Associated to a projection (5.14) for each $E_{k}$, there corresponds a toric crepant resolution $\hat{S}_{A_{r}(4)}$ of $S_{A_{r}(4)}$ with

$$
\chi\left(\hat{S}_{A_{r}(4)}\right)=\left|A_{r}(4)\right|=(r+1)^{3} .
$$

Furthermore, any two such $\hat{S}_{A_{r}(4)}$ differ by flops of 4-fold.

One can also describe a monomial basis of the $A_{r}(4)$-module $\mathbb{C}[Z] / I(x)$ for $x \in$ Hilb $^{A_{r}(4)}\left(\mathbb{C}^{4}\right)$, similar to the one we have given in Section 5 for the case $r=1$.

Another type of hypersurface orbifolds are those from the quotient singularities of simple groups. For $n=3$, the well-known examples are $S_{G}$ for $G=$ the icosahedral group $I_{60}$, Klein group $H_{168}$, in which cases a crepant resolution of $S_{G}$ was explicitly constructed in $[15,25]$, respectively. The structure of $\operatorname{Hilb}^{G}\left(\mathbb{C}^{3}\right)$ has recently been discussed in [6]. Even though the crepant and smooth property of the group orbit Hilbert schemes for dimension 3 is known by [1], a clear quantitative and qualitative relation would still be interesting for the possible study of some other simple groups 
$G$ in higher dimensions. Such program is under consideration with initial progress being made.

Even for the abelian group $G$ in the dimension $n=3$, the conclusion on the trivial canonical bundle of $\mathrm{Hilb}^{G}\left(\mathbb{C}^{3}\right)$ would raise a subtle question in the Mirror problem of Calabi-Yau 3-folds in string theory. As an example, a standard well-known one is the Fermat quintic in $\mathbb{P}^{4}$ with the special marginal deformation family

$$
X: \sum_{j=1}^{5} z_{i}^{5}+\lambda z_{1} z_{2} z_{3} z_{4} z_{5}=0, \quad \lambda \in \mathbb{C} .
$$

With the maximal diagonal group $S D$ of $z_{i}$ 's preserving the family $X$, the mirror $X^{*}$ is constructed by "the" crepant resolution of $X / S D, X^{*}=\widehat{X / S D}$ (cf. [8, 23]), by which the roles of $H^{1,1}, H^{2,1}$ are interchangeable in the "quantum" sense. When working on the one-dimensional space $H^{1,1}(X) \sim H^{2,1}\left(X^{*}\right)$, the choice of crepant resolution $\widehat{X / S D}$ makes no difference on the conclusion. While on the part of $H^{2,1}(X) \sim H^{1,1}\left(X^{*}\right)$, it has been known that many topological invariants, like Euler characteristic, Hodge numbers, elliptic genus, are independent of the choices of crepant resolutions, hence one obtains the same invariants for different choices of crepant resolutions as the model for $X^{*}$. However, the topological triple intersection of cohomologies does differ for two crepant resolutions (cf. [24]), hence the choice of crepant resolution as the mirror $X^{*}=\widehat{X / S D}$ will lead to the different effect on the topological cubic form of $H^{1,1}\left(X^{*}\right)$, upon which as the "classical" level, the quantum triple product of the physical mirror theory would be built (cf. [27]). The question of the "good" model for $X^{*}$ has rarely been raised in the past, partly due to the lack of mathematical knowledge on the issue. However, with the $G$-Hilbert scheme now given in Sections 3 and 4 as the mirror $X^{*}$, it seems to have left some fundamental open problems on its formalism of mirror Calabi-Yau spaces and the question of the arbitrariness of the choice of crepant resolutions remains a mathematical question to be completely understood concerning its applicable physical theory.

For the role of $G$-Hilbert scheme in the study of crepant resolution of $S_{G}$, the conclusion we have obtained for $G=A_{r}(4)$ has indicated that $\operatorname{Hilb}^{G}\left(\mathbb{C}^{n}\right)$ could not be a crepant resolution of $S_{G}$ in general when the dimension $n$ is greater than 3. Nevertheless the structure of $\operatorname{Hilb}^{G}\left(\mathbb{C}^{n}\right)$ is worthwhile for further study on its own right due to the interplay of geometry and group representations. Its understanding could still lead to the construction of crepant resolutions of $S_{G}$ in case such one does exist. It would be a promising direction of the geometrical study of orbifolds.

ACKNOWLEDGEMENTS. We wish to thank I. Nakamura for making us aware of his work $[6,19]$. This work is supported in part by the NSC of Taiwan under grant No. 89-2115-M-001-012.

\section{REFERENCES}

[1] T. Bridgeland, A. King, and M. Reid, The McKay correspondence as an equivalence of derived categories, J. Amer. Math. Soc. 14 (2001), no. 3, 535-554. CMP 1824990. Zbl 992.27561.

[2] L. Chiang and S. S. Roan, On hypersurface quotient singularity of dimension 4, http://xxx.lanl.gov/abs/math.AG/0011151. 
[3] D. Cox, J. Little, and D. O'Shea, Ideals, Varieties, and Algorithms. An Introduction to Computational Algebraic Geometry and Commutative Algebra, Undergraduate Texts in Mathematics, Springer-Verlag, New York, 1992. MR 93j:13031. Zbl 756.13017.

[4] V. I. Danilov, The geometry of toric varieties, Russian Math. Surveys 33 (1978), no. 2, 97-154, [translated from Uspekhi Mat. Nauk 33 (1978) 85-134. MR 80g:14001]. Zbl 425.14013.

[5] L. Dixon, J. A. Harvey, C. Vafa, and E. Witten, Strings on orbifolds, Nuclear Phys. B 261 (1985), no. 4, 678-686. MR 87k:81104a.

[6] Y. Gomi, I. Nakamura, and K. Shinoda, Hilbert schemes of G-orbits in dimension three, Asian J. Math. 4 (2000), no. 1, 51-70. CMP 1802 912. Zbl 992.22309.

[7] G. Gonzalez-Sprinberg and J.-L. Verdier, Construction géométrique de la correspondance de McKay, Ann. Sci. École Norm. Sup. (4) 16 (1983), no. 3, 409-449 (1984) (French). MR 85k:14019. Zbl 538.14033.

[8] B. R. Greene and M. R. Plesser, Duality in Calabi-Yau moduli space, Nuclear Phys. B 338 (1990), no. 1, 15-37. MR 91h:32018.

[9] F. Hirzebruch and T. Höfer, On the Euler number of an orbifold, Math. Ann. 286 (1990), no. 1-3, 255-260. MR 91g:57038. Zbl 679.14006.

[10] Y. Ito and H. Nakajima, McKay correspondence and Hilbert schemes in dimension three, Topology 39 (2000), no. 6, 1155-1191. MR 1783 852. Zbl 992.05914.

[11] Y. Ito and I. Nakamura, McKay correspondence and Hilbert schemes, Proc. Japan Acad. Ser. A Math. Sci. 72 (1996), no. 7, 135-138. MR 97k:14003. Zbl 881.14002.

[12] _ Hilbert schemes and simple singularities, New Trends in Algebraic Geometry (Warwick, 1996), London Math. Soc. Lecture Note Ser., vol. 264, Cambridge Univ. Press, Cambridge, 1999, pp. 151-233. MR 2000i:14004. Zbl 954.14001.

[13] G. Kempf, Finn Faye Knudsen, D. Mumford, and B. Saint-Donat, Toroidal Embeddings. I, Lecture Notes in Mathematics, vol. 339, Springer-Verlag, Berlin, 1973. MR 49\#299. Zbl 271.14017.

[14] F. Klein, Gesammelte mathematische Abhandlungen. II, Springer-Verlag, 1922 (German).

[15] D. Markushevich, Resolution of $\mathbf{C}^{3} / H_{168}$, Math. Ann. 308 (1997), no. 2, 279-289. MR 98i:14019. Zbl 899.14016.

[16] J. McKay, Graphs, singularities, and finite groups, The Santa Cruz Conference on Finite Groups (Univ. California, Santa Cruz, Calif., 1979) (Rhode Island), Proc. Sympos. Pure Math, vol. 37, Amer. Math. Soc., 1980, pp. 183-186. MR 82e:20014. Zbl 451.05026.

[17] S. Mori, Birational classification of algebraic threefolds, Proceedings of the International Congress of Mathematicians (Kyoto, 1990) (Tokyo), Math. Soc. Japan, 1991, pp. 235-248. MR 93b:14031. Zbl 751.14026.

[18] I. Nakamura, Hilbert schemes and simple singularities $E_{6}, E_{7}$ and $E_{8}$, preprint, 1996.

[19] , Hilbert schemes of abelian group orbits, J. Algebraic Geom. 10 (2001), no. 4, 757779. CMP 1838978.

[20] T. Oda, Torus Embeddings and Applications, Tata Institute of Fundamental Research Lectures on Mathematics and Physics, vol. 57, Tata Institute of Fundamental Research, Bombay, 1978. MR 81e:14001. Zbl 417.14043.

[21] S. S. Roan, On the generalization of Kummer surfaces, J. Differential Geom. 30 (1989), no. 2, 523-537. MR 90j:32032. Zbl 672.14024.

[22] _ On Calabi-Yau orbifolds in weighted projective spaces, Internat. J. Math. 1 (1990), no. 2, 211-232. MR 91m:14064. Zbl 793.14031.

[23] _ The mirror of Calabi-Yau orbifold, Internat. J. Math. 2 (1991), no. 4, 439-455. MR 92f:14037. Zbl 817.14018.

[24] _ Topological coupling of Calabi-Yau orbifold, J. Group Theory Phys. 1 (1993), 83103.

[25] _ On $c_{1}=0$ resolution of quotient singularity, Internat. J. Math. 5 (1994), no. 4, 523-536. MR 95g:14019. Zbl 856.14005. 

, Minimal resolutions of Gorenstein orbifolds in dimension three, Topology 35 (1996), no. 2, 489-508. MR 97c:14013. Zbl 872.14034.

[27] S.-T. Yau (ed.), Essays on Mirror Manifolds, International Press, Hong Kong, 1992. MR 94b:32001. Zbl 816.00010.

Li Chiang: Institute of Mathematics, Academia Sinica, TAipei, TAiwan

E-mail address: chiang1@gate. sinica.edu.tw

SHI-SHyr RoAn: InStitute of MATHEMATICS, ACADEMIA SinicA, TAIPEI, TAIWAN

E-mail address: maroan@ccvax. sinica.edu. tw 


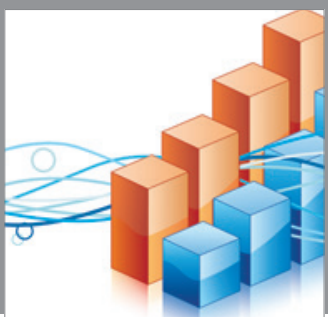

Advances in

Operations Research

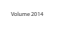

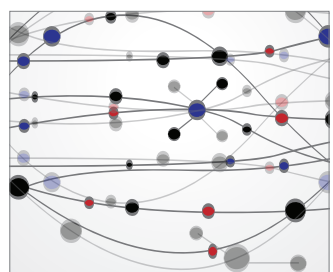

\section{The Scientific} World Journal
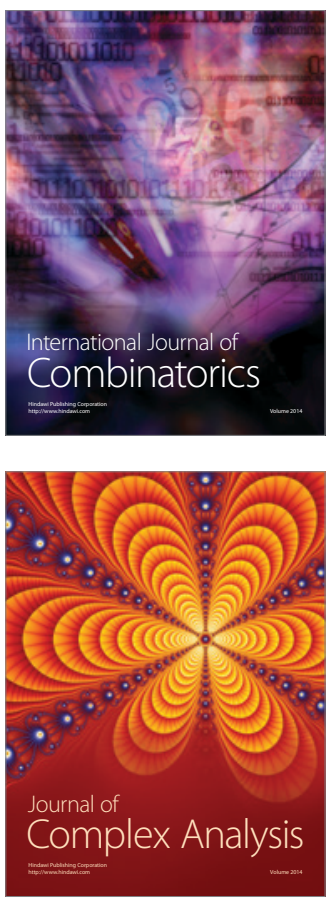

International Journal of

Mathematics and

Mathematical

Sciences
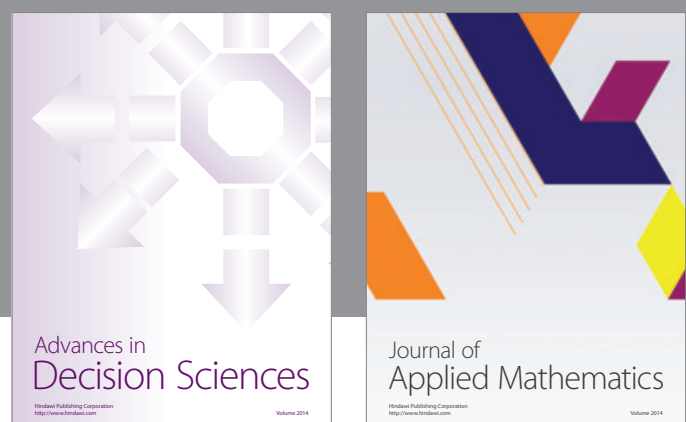

Journal of

Applied Mathematics
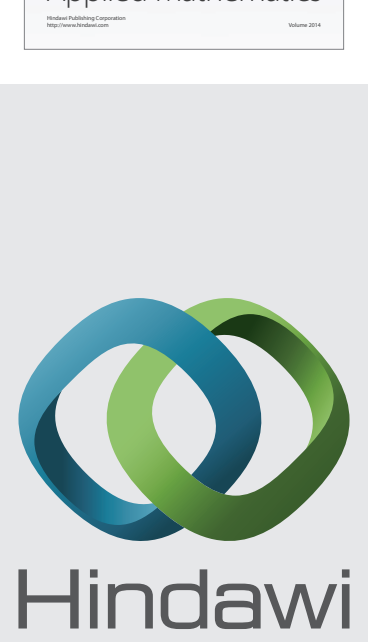

Submit your manuscripts at http://www.hindawi.com
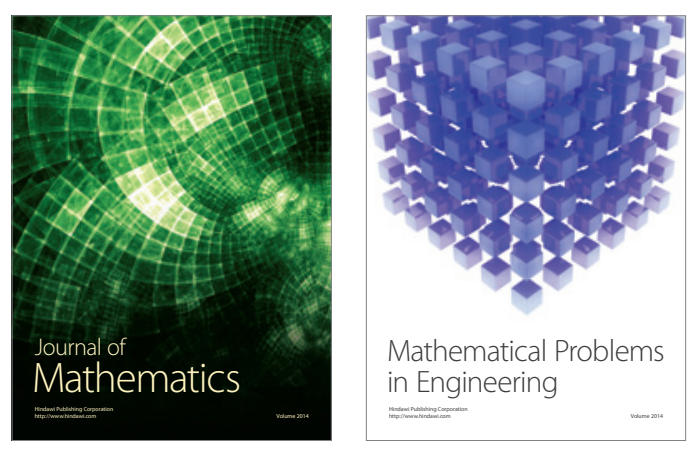

Mathematical Problems in Engineering
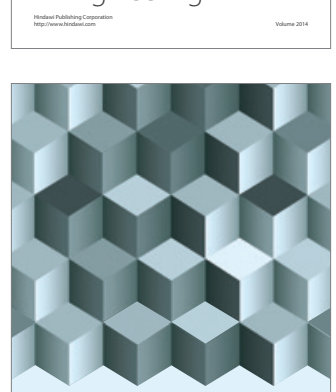

Journal of

Function Spaces
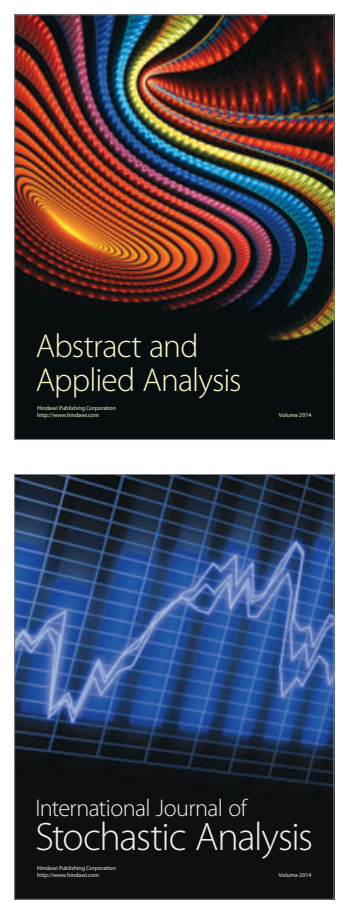

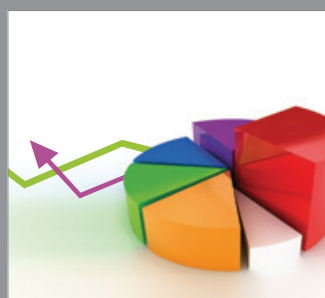

ournal of

Probability and Statistics

Promensencen
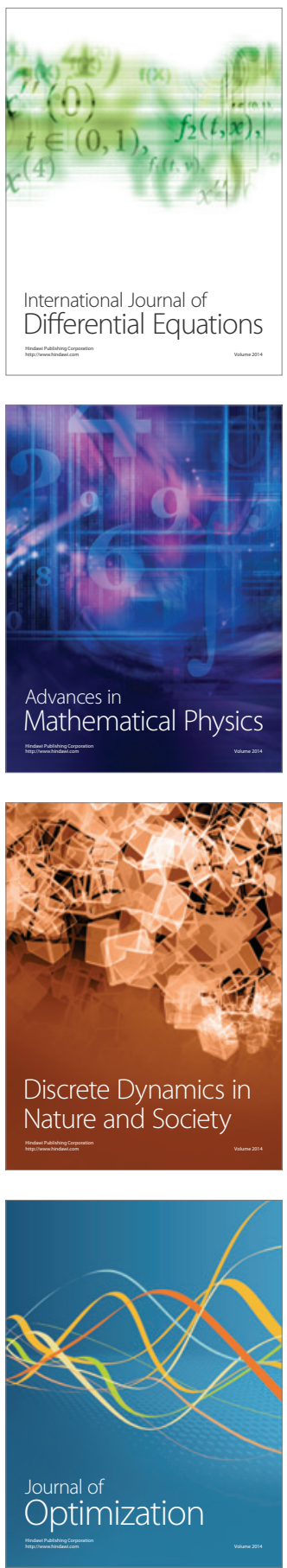